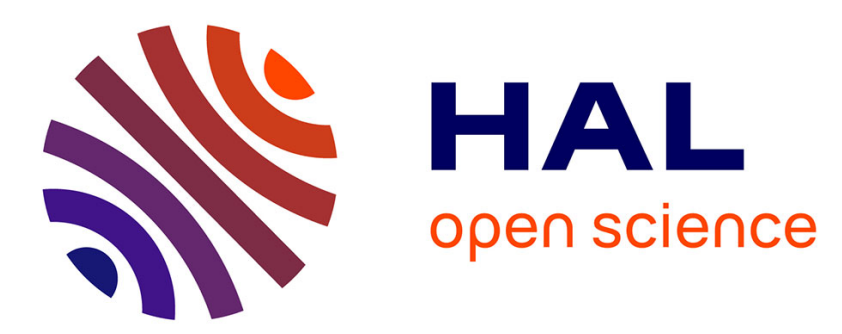

\title{
Magneto-optical observation of a magnetically induced " domain spin-flop " in a cubic antiferromagnet : KNiF3
}

\author{
R.H. Petit, J. Ferré, J. Nouet
}

\section{To cite this version:}

R.H. Petit, J. Ferré, J. Nouet. Magneto-optical observation of a magnetically induced " domain spin-flop " in a cubic antiferromagnet: KNiF3. Journal de Physique, 1975, 36 (5), pp.431-436. 10.1051/jphys:01975003605043100 . jpa-00208269

\section{HAL Id: jpa-00208269 https://hal.science/jpa-00208269}

Submitted on 1 Jan 1975

HAL is a multi-disciplinary open access archive for the deposit and dissemination of scientific research documents, whether they are published or not. The documents may come from teaching and research institutions in France or abroad, or from public or private research centers.
L'archive ouverte pluridisciplinaire HAL, est destinée au dépôt et à la diffusion de documents scientifiques de niveau recherche, publiés ou non, émanant des établissements d'enseignement et de recherche français ou étrangers, des laboratoires publics ou privés. 


\title{
MAGNETO-OPTICAL OBSERVATION \\ OF A MAGNETICALLY INDUCED « DOMAIN SPIN-FLOP » IN A CUBIC ANTIFERROMAGNET : $\mathrm{KNiF}_{3}$
}

\author{
R. H. PETIT, J. FERRÉ \\ Laboratoire d'Optique Physique (*), EPCI, 10, rue Vauquelin, 75231 Paris Cedex 05, France \\ and \\ J. NOUET
}

Faculté des Sciences, route de Laval, 72000 Le Mans, France

(Reçu le 6 décembre 1974, accepté le 16 janvier 1975)

\begin{abstract}
Résumé. - Sous faible champ magnétique $H$ nous avons observé une variation importante de l'allure des spectres magnétooptiques en fonction de $H$, pour un composé antiferromagnétique cubique : $\mathrm{KNiF}_{3}$. Ce phénomène, examiné ici pour la première fois, est comparé à l'apparition de la phase oblique, bien connue dans les composés antiferromagnétiques uniaxes. Des calculs spectroscopiques nous ont permis d'interpréter le spectre de dichroïsme circulaire magnétique observé de part et d'autre d'un champ critique $H_{\mathrm{cr}}$ au-delà duquel il ne subsiste que la phase oblique. La faible valeur de $H_{\mathrm{cr}}$ nous a conduit à montrer que le déplacement réversible des parois de mâcle précède la rotation des spins dans un monodomaine.
\end{abstract}

\begin{abstract}
We have observed a large change in the field dependence of the magneto-optical spectra of cubic $\mathrm{KNiF}_{3}$ at a low magnetic field. This phenomenon, which is being reported for the first time, is compared to the well-known spin-flop occurring in uniaxial antiferromagnets. Spectroscopic calculations allow us to attribute the magnetic circular dichroism signals that have been observed below and above the critical field, to an antiferromagnetic and a spin-flop phase. We verify that in a cubic antiferromagnet, the reversible movement of domain twin-walls occurs at a lower magnetic field than the rotation of the spins inside a single domain.
\end{abstract}

1. Introduction. - The spin-flop phenomenon of sublattices of a uniaxial two sublattice antiferromagnet is well known [1]. It occurs when the magnetic field applied along the easy axis exceeds a critical value $H_{\mathrm{SF}}=\sqrt{2 H_{\mathrm{A}} H_{\mathrm{E}}}$, where $H_{\mathrm{A}}$ and $H_{\mathrm{E}}$ are the anisotropy and the exchange fields respectively. Then, in a finite interval of field near $H_{\mathrm{SF}}$, the antiferromagnetic vector $\mathbf{l}=\mathbf{m}_{1}-\mathbf{m}_{2}$ turns from the direction of the easy axis (antiferromagnetic AF state) to that perpendicular to it and since the spins tilt slightly towards the field direction there appears a net magnetisation $\mathbf{m}=\mathbf{m}_{1}+\mathbf{m}_{2}$ (spin-flop SF state) $[2-4]$.

In a cubic antiferromagnet such as $\mathrm{KNiF}_{3}$, which remains cubic below $T_{\mathrm{N}}=246 \mathrm{~K}$ [5], the situation differs markedly. We must take account of the fact that in the absence of an external magnetic field, we generally start from a multidomain structure.

(*) Equipe de recherches $n^{\circ} 5$ du C.N.R.S.
Three kinds of domains must exist in which $\mathbf{l}$ is parallel to one of the fourfold axes [5-6]. The domain structure is sample dependent because it is essentially related to local imperfections associated with internal strains. Twin-walls separate such domains [7] and can move easily under stress or magnetic perturbations. When increasing the magnetic field applied along the fourfold axis $z$ a transition occurs at a low field. In our attempt to give an interpretation of this transition, we demonstrate here that it is not due to a microscopic phenomenon which involves $H_{\mathrm{A}}$ and $H_{\mathrm{E}}$ but rather to a reversible movement of twinwalls at a low magnetic field, this movement being related to the transformation of $d_{z}$ domains into $d_{x}$ or $d_{y}$ domains. This agrees with suggestions made earlier by Néel [8]. We name this transition domain spin-flop (DSF).

A spectroscopic study of the intermediate state was necessary to demonstrate the coexistence of the two AF and SF phases for an uniaxial antiferro- 
magnet [3-4]. In our case, the absorption is only slightly modified at the DSF and we have therefore used a more sensitive method, i.e. magnetic circular dichroism (MCD).

The apparatus described earlier [9] is able to detect dichroism of about $10^{-6}$ in absorbance. Here the field is provided by a superconducting solenoid which gives an homogeneity better than one per cent.

2. Evidence for AF and SF phases by MCD. 2.1 ON EXCITONIC TRANSITIONS. - 2.1.1 Antiferromagnetic phase : $d_{z}$ domains. - First, we consider only $d_{z}$ domains. We suppose that when we apply a weak magnetic field along $z$, the spins of the $a$ and $b$ sublattices remain oriented antiferromagnetically and parallel to $z$.

We can write the wave function $|G\rangle$ of the ground state of the entire crystal [10]

$$
|G\rangle=A \prod_{n=1}^{N}\left|{ }^{3} \mathrm{~A}_{2}+1\right\rangle_{n a} \prod_{m=1}^{N}\left|{ }^{3} \mathrm{~A}_{2}-1\right\rangle_{m b}
$$

$n$ and $m$ refer to the unit cells in the two sublattices. $A$ is the antisymmetrisation operator. Note that the spins are quantised along the $z$ axis.

The exciton corresponding to the electronic transition ${ }^{3} \mathrm{~A}_{2 \mathrm{~g}} \rightarrow{ }^{1} \mathrm{E}_{\mathrm{g}}$ in the sublattice $\mu$ ( $a$ or $b$ ), for the wave vector $\mathbf{k}$ is

$$
\left|E^{\mu}, \mathbf{k}\right\rangle=N^{-1 / 2} \sum_{n=1}^{N} \exp \left(\mathbf{i k . r _ { n }}\right)|E n \mu\rangle
$$

if we suppose that the intersublattice interaction is negligible.

Here $|E n \mu\rangle$ stands for the excited state in which the $n \mu$ th ion is excited.

$$
\begin{aligned}
|E n a\rangle_{\alpha}=A\left|{ }^{1} \mathrm{E}_{\mathrm{g}} \alpha\right\rangle_{n a} \prod_{j \neq n}\left|{ }^{3} \mathrm{~A}_{2}+1\right\rangle_{j a} \times \\
\times \prod_{m=1}^{N}\left|{ }^{3} \mathrm{~A}_{2}-1\right\rangle_{m b} \\
|E m b\rangle_{\alpha}=A\left|{ }^{1} \mathrm{E}_{\mathrm{g}} \alpha\right\rangle_{m b} \prod_{j \neq m}\left|{ }^{3} \mathrm{~A}_{2}-1\right\rangle_{j b} \times \\
\times \prod_{n=1}^{N}\left|{ }^{3} \mathrm{~A}_{2}+1\right\rangle_{n a}
\end{aligned}
$$

$\alpha$ is the component $\varepsilon$ or $\theta$ of ${ }^{1} \mathrm{E}_{\mathrm{g}}$ in the $\mathrm{O}_{\mathrm{h}}$ group. In order to calculate the $\mathrm{MCD}$, we must consider the components $\beta= \pm 1$ of the magnetic dipolar moment operator $\hat{m}$.

For the excitonic transition, $\mathbf{k}=0$, then :

$$
\begin{aligned}
& \left\langle E_{\alpha}^{a}, \mathbf{k}=0\left|\hat{m}_{\beta}\right| G\right\rangle=\left\langle{ }^{1} \mathrm{E}_{\mathrm{g}} \alpha\left|\hat{m}_{\beta}\right|{ }^{3} \mathrm{~A}_{2}+1\right\rangle \\
& \left\langle E_{\alpha}^{b}, \mathbf{k}=0\left|\hat{m}_{\beta}\right| G\right\rangle=\left\langle{ }^{1} \mathrm{E}_{\mathrm{g}} \alpha\left|\hat{m}_{\beta}\right|{ }^{3} \mathrm{~A}_{2}-1\right\rangle .
\end{aligned}
$$

By considering the second member of the above expressions, we deduce that $\sigma_{+}$polarised light in the $a$ sublattice corresponds to $\sigma_{-}$polarised light with the same intensity in the $b$ sublattice. Then the observed MCD in a low magnetic field arises from the difference in energy between the up-spin and down-spin sublattices, i.e. $2 g \mu_{\mathrm{B}} H\left(g=\right.$ Landé factor of ${ }^{3} \mathrm{~A}_{2 \mathrm{~g}}, \mu_{\mathrm{B}}=\mathrm{Bohr}$ magneton). For an isolated transition which gives rise to an absorption line (bandwidth $\gamma$ ) and when $g \mu_{\mathrm{B}} H \ll \gamma$, the MCD $\Delta A_{\mathrm{c}}(\sigma)$ looks like the first derivative of the absorption curve, i.e. $\mathrm{d} f(\sigma) / \mathrm{d} \sigma[11]$. In this case, we shall speak of a $\mathrm{d} f(\sigma) / \mathrm{d} \sigma$ term. Above the DSF, the $\mathrm{d} f(\sigma) / \mathrm{d} \sigma$ term vanishes because the magnetic energy of the two sublattices is identical.

2.1.2 Spin-flop phase : $d_{x}$ and $d_{y}$ domains. For $\mathrm{KNiF}_{3}$, inside $d_{x}$ and $d_{y}$ domains the spins tend to align themselves along the field direction. Then the spin configuration is the same as in the spin-flop phase of a uniaxial antiferromagnet. So that the method of our calculations is valid whatever the crystal symmetry.

We shall use the Wigner functions for spin rotation in order to write the wave functions of the entire crystal under a magnetic field. We keep $z$ as the quantisation axis, and consider that under the magnetic field, the spins of the $a$ (or $b$ ) sublattice deviate through the angle $\pi-\theta$ (or $\theta$ ) from the $z$ direction in the ground state of the crystal $\left(\cos \theta=H / 2 H_{\mathrm{E}}\right)$.

Then

$$
|G\rangle^{\prime}=A \prod_{n=1}^{N}\left|{ }^{3} \mathrm{~A}_{2}+1\right\rangle_{n a}^{\prime} \prod_{m=1}^{N}\left|{ }^{3} \mathrm{~A}_{2}-1\right\rangle_{m b}^{\prime}
$$

stands for the ground state of the crystal, with :

$$
\begin{aligned}
& \left|{ }^{3} \mathrm{~A}_{2}+1\right\rangle_{n a}^{\prime}=\sin ^{2} \frac{\theta}{2}\left|{ }^{3} \mathrm{~A}_{2}+1\right\rangle_{n a} \\
& +\sqrt{2} \cos \frac{\theta}{2} \sin \frac{\theta}{2}\left|{ }^{3} \mathrm{~A}_{2} 0\right\rangle_{n a} \\
& +\cos ^{2} \frac{\theta}{2}\left|{ }^{3} \mathrm{~A}_{2}-1\right\rangle_{n a} \\
& \left|{ }^{3} \mathrm{~A}_{2}-1\right\rangle_{m b}^{\prime}=\sin ^{2} \frac{\theta}{2}\left|{ }^{3} \mathrm{~A}_{2}+1\right\rangle_{m b} \\
& -\sqrt{2} \cos \frac{\theta}{2} \sin \frac{\theta}{2}\left|{ }^{3} \mathrm{~A}_{2} 0\right\rangle_{m b} \\
& +\cos ^{2} \frac{\theta}{2}\left|{ }^{3} \mathrm{~A}_{2}-1\right\rangle_{m b} \text {. }
\end{aligned}
$$

The excited state $\left|{ }^{1} \mathrm{E}_{\mathrm{g}}\right\rangle_{n \mu}$ of the $n \mu$ th ion is not modified by a spin rotation because its spin equals zero.

The exciton $\left|E^{\mu}, \mathbf{k}\right\rangle^{\prime}$ is obtained by replacing $\left|{ }^{3} \mathrm{~A}_{2}+1\right\rangle_{n a}$ and $\left|{ }^{3} \mathrm{~A}_{2}-1\right\rangle_{m b}$ by $\left|{ }^{3} \mathrm{~A}_{2}+1\right\rangle_{n a}^{\prime}$ and $\left|{ }^{3} A_{2}-1\right\rangle_{m b}^{\prime}$ in the expression (2.2). Here we keep the same angle $\theta$ in the exciton wave function as in the ground state. We should have taken $\theta^{\prime}$ depending upon the exchange between an excited ion and surrounding ground state ions while $\theta$ depends only upon the exchange in the ground state. Therefore, we are assuming that the excitation of one ion does not modify the spins of the others. 
Petrov [12] has demonstrated that the spin-flop phase is characterised by the removal of the degeneracy between the excitonic levels

$$
\left|E^{ \pm}, \mathbf{k}\right\rangle=(1 / \sqrt{2})\left(\left|E^{a}, \mathbf{k}\right\rangle^{\prime} \pm\left|E^{b}, \mathbf{k}\right\rangle^{\prime}\right) \text {. }
$$

The splitting, which is called the Davidov splitting at $\mathbf{k}=0$, is proportional to the energy of transfer of the excitation to ${ }^{1} \mathrm{E}_{\mathrm{g}}$ from one sublattice to the other; it depends upon the exchange interaction and is proportional to $\cos ^{2} \theta$ [12].

As in the case of the antiferromagnetic phase, we have calculated the MCD for the excitonic transitions $\left(\left|E^{ \pm} \varepsilon\right\rangle\right.$ and $\left.\left|E^{ \pm} \theta\right\rangle\right)$. For $\left|E^{-} \varepsilon\right\rangle$ and $\left|E^{-} \theta\right\rangle$ the MCD is zero. For $\left|E^{+} \varepsilon, \theta\right\rangle$ we find an MCD term proportional to the absorption curve $f(\sigma)$, the amplitude of which is :

$$
\begin{aligned}
& \Delta A_{\mathrm{c}}=\left(\frac{2}{3}\right) m_{0}^{2} \cos \theta, \text { for }\left|E^{+} \varepsilon\right\rangle \\
& \Delta A_{\mathrm{c}}=-2 m_{0}^{2} \cos \theta, \text { for the exciton }\left|E^{+} \theta\right\rangle,
\end{aligned}
$$

where $m_{0}$ is proportional to the reduced matrix element of the magnetic dipole moment

$$
\left\langle{ }^{1} \mathrm{E}_{\mathbf{g}}|| \hat{m}||^{3} \mathrm{~A}_{2 \mathrm{~g}}\right\rangle \text {. }
$$

The exchange interaction, the spin orbit coupling and the applied magnetic field do not lift the degeneracy of ${ }^{1} \mathrm{E}_{\mathrm{g}} \varepsilon$ and ${ }^{1} \mathrm{E}_{\mathrm{g}} \theta$ levels. Then for the pure excitonic transition we obtain $\Delta A_{\mathrm{c}} / A_{m}=-\cos \theta$ where $A_{m}=4 m_{0}^{2} / 3$ stands for the maximum absorbance of the line in the absence of the magnetic field.

2.2 ON EXCITON-PHONON TRANSITIONS. - We now consider the exciton-phonon transitions induced by one odd parity phonon. We make the hypothesis that the MCD and absorption spectra can be interpreted by considering that the phonons $t_{1 u}$ at $\mathbf{k}=0$ are the most influential [13]. Then it is possible to estimate the MCD in the antiferromagnetic [14] and in the spin-flop phases. For the latter case, in the same way as for the exciton transition, we calculate $\Delta A_{\mathrm{c}}(\sigma) / A_{\mathrm{m}}=\frac{1}{2} \cos \theta \cdot f(\sigma)$ for the exciton-one phonon $\mathrm{t}_{1 \mathrm{u}}$ transition.

It is interesting to compare our results to those of a pure ferromagnetic phase where

$$
|G\rangle=\prod_{n=1}^{N}\left|{ }^{3} \mathrm{~A}_{2}-1\right\rangle_{n}
$$

We deduce the MCD for the zero-phonon transition : $\Delta A_{\mathrm{c}}(\sigma) / A_{\mathrm{m}}=(-1) f(\sigma)$ and for the exciton-one phonon $\mathrm{t}_{1 \mathrm{u}}$ at $\mathrm{k}=0: \Delta A_{\mathrm{c}}(\sigma) / A_{\mathrm{m}}=\frac{1}{2} f(\sigma)$. We note that in the spin-flop phase or for $d_{x}$ and $d_{y}$ domains, the MCD is only due to the ferromagnetic component.

2.3 GeNerAl EXPRESSION FOR MCD. - In conclusion, the MCD allows us to separate the effects due to $d_{z}$ domains from those due to $d_{x}$ and $d_{y}$ domains. In an ideal case and without perturbation, three kinds of domains $\left(d_{x}, d_{y}, d_{z}\right)$ must exist. A magnetic field $H_{z}$ favours $d_{x}$ and $d_{y}$ domains.
We have demonstrated that, if $N_{z}$ represents the proportion of the $d_{z}$ domains, the MCD is expressed by :

$$
\frac{\Delta A_{\mathrm{c}}(\sigma)}{A_{\mathrm{m}}}=H\left[N_{z} a \mu_{\mathrm{B}} \frac{\mathrm{d} f(\sigma)}{\mathrm{d} \sigma}+\left(1-N_{z}\right) c f(\sigma)\right]
$$

in the rigid shift approximation. The $a$ and $c$ coefficients (Table I) depend on the optical transition.

\section{TABLE I}

$a$ and $c$ theoretical values for excitonic and exciton-phonon $\left(\mathrm{t}_{1 \mathrm{u}}\right.$ at $\left.\mathrm{k}=0\right){ }^{3} \mathrm{~A}_{2 \mathrm{~g}} \rightarrow{ }^{1} \mathrm{E}_{\mathrm{g}}$ transition

$\begin{array}{cccc}\text { Coefficient } & \begin{array}{c}\text { Excitonic } \\ \text { transition }\end{array} & \begin{array}{c}\text { Exciton-phonon } \\ \left(\mathrm{t}_{1 \mathrm{u}}\right) \text { transition }\end{array} \\ - & - & - & - \\ a & & +g & -\frac{g}{2} \\ & \left|E^{+}\right\rangle & -\frac{1}{2 H_{\mathrm{E}}} & \frac{1}{4 H_{\mathrm{E}}} \\ c & \left|E^{-}\right\rangle & 0 & 0\end{array}$

2.4 EXPERIMENTAL RESULTS. - 2.4.1 General considerations. - The MCD signals may be altered by linear magnetic birefringence and linear dichroism due to the inequivalency of the number of $d_{x}$ and $d_{y}$ domains.

Such a stray magnetic birefringence depends upon the quality and the thickness of the crystal and also upon the orientation of its fourfold axis $z$ with respect to the light and the magnetic field directions. Small internal strains of several $\mathrm{kg} / \mathrm{cm}^{2}$ oriented along one of the $x$ or $y$ axes, lead to a large linear dichroism [5]. The most reliable spectra are obtained for crystals with only a few imperfections, impurities and internal strains.

We have obtained the spectra for a thin crystal $(e=0.195 \mathrm{~mm})$ of good quality. Before each experiment, we verified that no linear dichroism appeared at low temperature and in order to be sure that we observed the real MCD, we have also verified that its sign reverses on reversing $\mathbf{H}$.

Note that we obtained better results when we cooled the sample in the absence of an applied field. This is consistent with the fact that near $T_{\mathrm{N}}$ the spins orient easily themselves perpendicular to a small magnetic field.

2.4.2 Field dependence of the spectra. - The spectra for the ${ }^{3} \mathrm{~A}_{2 \mathrm{~g}} \rightarrow{ }^{1} \mathrm{E}_{\mathrm{g}}$ transition are given in figure 1 . Note that the DSF has been observed for all optical transitions but the ${ }^{3} \mathrm{~A}_{2 \mathrm{~g}} \rightarrow{ }^{1} \mathrm{E}_{\mathrm{g}}$ transition has been chosen because of the simplicity of the excited state.

We observe MCD (Fig. 1) temperature independent terms $(5 \mathrm{~K}<T<25 \mathrm{~K})$ proportional to $\mathrm{d} f(\sigma) / \mathrm{d} \sigma$ for low magnetic fields and to $f(\sigma)$ for high fields $(H=37 \mathrm{kOe})$, on the first absorption peaks which are well isolated in the spectrum. The high field spectrum is consistent with the MCD shape dispersion 


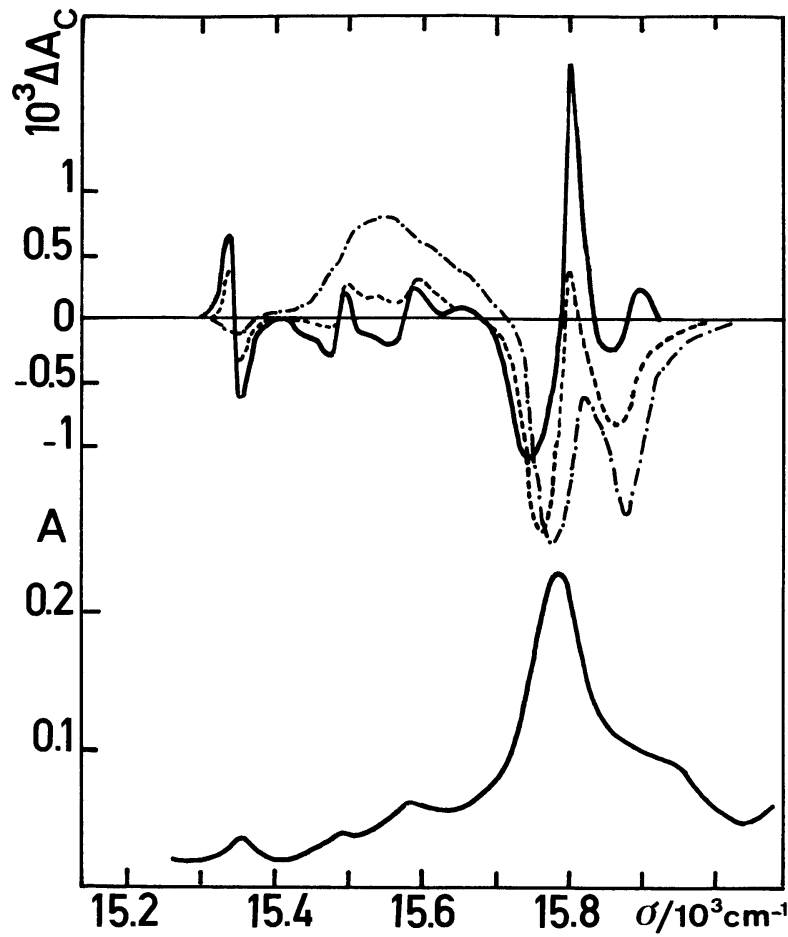

Fig. 1. - Absorption at $H=0$ (bottom) and MCD spectra (top) for the ${ }^{3} \mathrm{~A}_{2 \mathrm{~g}} \rightarrow{ }^{1} \mathrm{E}_{\mathrm{g}}^{\mathrm{a}}$ transition in $\mathrm{KNiF}_{3}$ at $15 \mathrm{~K}$. We have shown the MCD spectra for $H=6.25 \mathrm{kOe}(\longrightarrow), H=19 \mathrm{kOe}(--)$, $H=37 \mathrm{kOe}(-.-)$. The thickness of the sample is $0.195 \mathrm{~mm}$. The bandwidth used is $3 \mathrm{~cm}^{-1}$.

for the ferrimagnetic $\mathrm{RbNiF}_{3}$ [13] where the ferromagnetic component aligns along the field.

Then we have followed the variation of MCD terms when slowly varying the magnetic field $(25 \mathrm{Oe} / \mathrm{s})$. The time constant used was $2.5 \mathrm{~s}$. As an example figure $2 a$ shows the variation of the extremum of a $\mathrm{d} f(\sigma) / \mathrm{d} \sigma$ term for the first absorption peak with field. Because of the smallness of the MCD signal at high field, we also give the field variation for the extremum of the $f(\sigma)$ term for a larger peak located at $15485 \mathrm{~cm}^{-1}$ (Fig. $2 b$ ).

In a magnetic field the spin inside $d_{x}$ or $d_{y}$ domains should tilt and give a $f(\sigma)$ signal. The absence of such a signal at low field indicates an almost $d_{z}$ type crystal. Between $12 \mathrm{kOe}$ and $20 \mathrm{kOe}$, the $\mathrm{d} f(\sigma) / \mathrm{d} \sigma$ term decreases and it reaches zero at $H_{\mathrm{cr}}=20 \mathrm{kOe}$ (Fig. 2a) meanwhile the $f(\sigma)$ term increases. This is consistent with the fact that the $d_{z}$ domains disappear at the expense of $d_{x}$ or $d_{y}$ domains. Between 12 and $20 \mathrm{kOe}$, the MCD behaviour (Fig. 1) indicates clearly the coexistence of all phases. Above $H_{\text {cr }}$ there only remain $d_{x}$ and $d_{y}$ domains. Since the linear dichroism is small, we conclude that they have practically equal importance.

At the same time we have observed a rapid variation of the absorption for all peaks around $15 \mathrm{kOe}$ : if we increase the magnetic field, the first absorption peak located at $15347 \mathrm{~cm}^{-1}$ decreases by about $20 \%$ and the others increase by only a few per cent, whatever the polarisation of the light. We cannot

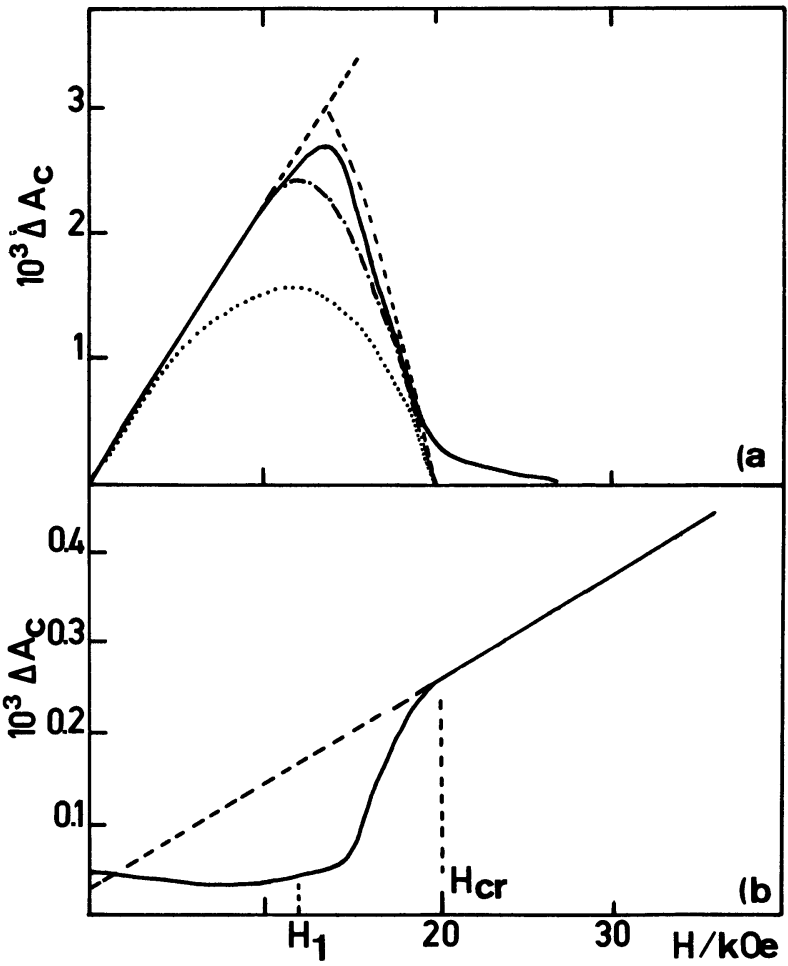

Fig. 2. - $-a$ ) Experimental ( $\longrightarrow$ ) and calculated field dependence of the peak to peak amplitude of the $\mathrm{d} f(\sigma) / \mathrm{d} \sigma \mathrm{MCD}$ term for the magnetic dipole ${ }^{3} \mathrm{~A}_{2 \mathrm{~g}} \rightarrow{ }^{1} \mathrm{E}_{\mathrm{g}}^{\mathrm{a}}$ transition $\left(\sigma_{0}=15347 \mathrm{~cm}^{-1}\right)$ for $H_{\text {cr }}=20 \mathrm{kOe}, H_{1}=0(\ldots), H_{1}=11.6 \mathrm{kOe}(-.-), H_{1}=14 \mathrm{kOe}$ $(--) ; b)$ Field dependence of the magnitude of the $f(\sigma)$ MCD term for the first exciton-phonon $\left(\mathrm{t}_{1 \mathrm{u}}\right)$ peak which is located at $15485 \mathrm{~cm}^{-1}$. For the $(a)$ and $(b)$ experimental curves the error does not exceed $\Delta A_{\mathrm{c}}=2 \times 10^{-5}$.

attribute this variation to birefringence and the associated linear dichroism because these have been verified to remain small $\left(\Delta A_{\mathrm{L}} / A_{\mathrm{m}}<10^{-3}\right)$. The absorption transition probabilities may thus be altered by the change in the site group symmetry from $\mathrm{C}_{4 \mathrm{~h}}$ to $\mathrm{C}_{2 \mathrm{~h}}$, related to the change in the magnetisation direction.

2.5 Discussion. - 2.5.1 At low magnetic field $(H<12 \mathrm{kOe})$. - The weak dipole strength of the first absorption peak located at $15347 \mathrm{~cm}^{-1}$ and the sign of its MCD $\mathrm{d} f(\sigma) / \mathrm{d} \sigma$ term under a magnetic field confirm its magnetic dipolar origin. From the $a$ value we can estimate $g=2.3 \pm 0.1$, which is close to $g=2.28$ predicted theoretically (Table I).

The energies of the other near absorption peaks, the sign and amplitude of the corresponding $a$ values [14], $a=1.1 \pm 0.2$, allow us to attribute them to $t_{1 u}$ phonon lines at $\mathbf{k}=0$ (Table $I$ ).

These results confirm that at low field almost all the spins are oriented along $z$.

2.5.2 Above the critical field $(20 \mathrm{kOe}) .-\mathrm{We}$ verify (Fig. 2) that the MCD varies linearly with $H$ above the transition, which confirms the fact that $\Delta A_{\mathrm{c}} / A_{\mathrm{m}}$ varies as $\cos \theta=H / 2 H_{\mathrm{E}}$. This fact supports our hypothesis $\theta=\theta^{\prime}$. If we had $\theta^{\prime} \neq \theta$ the MCD would be multiplied by new terms depending on $H$, 
such as the overlap of the wavefunctions of the ions which are not excited. For example

$$
\left\langle{ }^{3} \mathrm{~A}_{2}+1(\theta) \mid{ }^{3} \mathrm{~A}_{2}+1\left(\theta^{\prime}\right)\right\rangle=\cos ^{2}\left[\left(\theta-\theta^{\prime}\right) / 2\right] .
$$

Moreover, we find the proper sign for the exciton line (negative dichroism) and for the two first excitonphonon $\left(\mathrm{t}_{1 \mathrm{u}}\right)$ lines (positive MCD). The experimental values of the corresponding $f(\sigma)$ terms are very small since $g \mu_{\mathrm{B}} H\left(1-5 \mathrm{~cm}^{-1}\right) \ll g \mu_{\mathrm{B}} H_{\mathrm{E}}$ which implies $\cos \theta$ to be very small. These values agree with those predicted theoretically by taking

$$
g \mu_{\mathrm{B}} H_{\mathrm{E}}=|J| z\langle S\rangle=420 \mathrm{~cm}^{-1} \quad[13] .
$$

Thus we verify that the appearence of $\mathrm{MCD}$ in the spin-flop phase is only related to the net ferromagnetic component $\mathbf{m}$.

3. Evidence of a twin-wall movement. - We remark that the phenomenon seems to be reversible when increasing or decreasing the magnetic field $(0-45 \mathrm{kOe})$. At low temperature $(6 \mathrm{~K})$ the sharpness of the transition is greater than at higher temperatures $(210 \mathrm{~K})$.

We give some evidence which argues against the hypothesis of a spin-flop transition similar to the phase transition in uniaxial antiferromagnets :

$-H_{\text {cr }}$ depends on the crystal shape and on its thickness and varies from 8 to $22 \mathrm{kOe}$ for our samples ( 0.2 to $14 \mathrm{~mm}$ ).

- We find the same $H_{\text {cr }}$ value for all temperatures between $6 \mathrm{~K}$ and $210 \mathrm{~K}$.

- Our $H_{\text {cr }}$ value does not correspond to the experimental one-magnon $(\mathbf{k}=0)$ Raman frequency : $4.5 \mathrm{~cm}^{-1}[15]$.

- No new transition appears at the theoretical spin-flop field at $4.5 \mathrm{~cm}^{-1}$ [15]. We have continued experiments up to $45.6 \mathrm{kOe}\left(4.84 \mathrm{~cm}^{-1}\right)$.

- As the MCD presents no varying noise at the transition, we deduce [16] that there is no spin fluctuation at the DSF.

Thus we are going to show that the reversible movement of twin-walls precedes the spin flopping of the spins inside the domains, when increasing the magnetic field in cubic antiferromagnets, as has been suggested by Néel [8].

So we want to explain theoretically the DSF. For a domain (with direction cosines of the spins $\alpha$, $\beta$, $\gamma$, with respect to the crystallographic axes), the free energy per unit volume due to the interaction with an applied magnetic field $\mathbf{H}$ (direction cosines $\mu$, $v, \rho)$ can be defined as [6] :

$$
F=\frac{1}{2}\left(\chi_{\perp}-\chi_{\|}\right) H^{2}(\alpha \mu+\beta v+\gamma \rho)^{2}+\frac{1}{2} \chi_{\perp} H^{2}
$$

where $\chi_{\perp}$ and $\chi_{\|}$stand for the perpendicular and parallel susceptibilities of the crystal.

Here $\mu=v=0, \rho=1$. We consider a displacement $x$ of the wall between a $d_{z}$ domain and $d_{x}$ or $d_{y}$ domains, which corresponds to a rotation of the spins from the $(\alpha=\beta=0, \gamma=1)$ to the $\left(\alpha^{\prime}, \beta^{\prime}\right.$, $\gamma^{\prime}=\cos \varphi$ ) direction. The variation of free energy per area unit due to the volume variation may be written as : $-\frac{1}{2} x\left(\chi_{\perp}-\chi_{\|}\right) H^{2} \sin ^{2} \varphi$.

The variation $\Delta F$ of the total energy, in which we must include the effect of the restoring force $k x^{2} / 2$, has been minimized with respect to $x$ and $\varphi$. We deduced

$x=\left[\left(\chi_{\perp}-\chi_{\|}\right) H^{2} \sin ^{2} \varphi\right] / 2 k$ and $\varphi=\frac{\pi}{2}+K \pi$.

These results indicate that the walls move gradually with $H$ and disappear when $x$ reaches the value $\mathcal{L}$ of the width of the $d_{z}$ domains, at a field

$$
H_{\text {cr }}=\sqrt{2 \mathrm{~kL} /\left(\chi_{\perp}-\chi_{\|}\right)} .
$$

This agrees with the gradual field dependence of the MCD terms (Fig. 2) between 12 and $20 \mathrm{kOe}$. This behaviour is very different from the brutal SF transition in a uniaxial antiferromagnet. The sample dependence of the $H_{\text {cr }}$ values is related to the dispersion of $k$ and $\mathcal{L}$ due to the poor-definition of the domains in cubic antiferromagnets.

We have looked more carefully at the field dependence of the MCD terms (Fig. 2). In the formula (2.5), $N_{z}$ is proportional to the width $(\mathfrak{L}-x)$ of the $d_{z}$ domains. Then we have compared the field dependence of the $\mathrm{d} f(\sigma) / \mathrm{d} \sigma$ term to the expression

$$
N_{z} H \sim H\left(1-H^{2} / H_{\mathrm{cr}}^{2}\right)
$$

(Fig. 2a) and that of the $f(\sigma)$ term to $H\left(H^{2} / H_{\mathrm{cr}}^{2}\right)$ (Fig. $2 b$ ). In figure $2 b$ we observe that there is no variation of the MCD term below $12 \mathrm{kOe}$, which disagrees with the $H\left(H^{2} / H_{\mathrm{cr}}^{2}\right)$ law. Thus, we have introduced phenomenologically a new term in the expression of $\Delta F: x B \sin ^{2} \varphi$ and we have deduced

$$
H_{1}=\sqrt{2 B /\left(\chi_{\perp}-\chi_{\|}\right)}
$$

and

$$
H_{\text {cr }}=\sqrt{2(B+k \complement) /\left(\chi_{\perp}-\chi_{\|}\right)} .
$$

For $H<H_{1}, x$ is zero and there is no change in the domains structure. For

$$
H>H_{1}, \quad x=\mathfrak{L}\left(H^{2}-H_{1}^{2}\right) /\left(H_{\mathrm{cr}}^{2}-H_{1}^{2}\right) .
$$

A comparison between the experimental and calculated values of the $\mathrm{d} f(\sigma) / \mathrm{d} \sigma$ term (Fig. $2 a$ ) has led us to estimate a threshold field $H_{1}=(12 \pm 2) \mathrm{kOe}$.

The threshold field value depends upon the sample and varies from 0 to $15 \mathrm{kOe}$ for our crystals. We have verified that the application of a stress along $z$, which favours $d_{z}$ domains [5], increases the $H_{1}$ value. Thus, we have concluded that $B$, which is small compared to the anisotropy energy of $\mathrm{KNiF}_{3}$, may be the difference of anisotropy between the $z$ and the $x$ or $y$ directions. 
4. Conclusion. - We have shown that MCD experiments allow us to follow accurately the spin-flop transition. The MCD is related in a simple way to the tilt angle $\theta$ between the magnetic moments for two opposite sublattices in $\mathrm{KNiF}_{3}$ under a magnetic field. In some antiferromagnets we may have the same configuration of a weak ferromagnetism, due to a Dzialoshinski exchange. Circular dichroism could be used in order to determine precisely the angle $\theta$ in these materials.

For the cubic antiferromagnet $\mathrm{KNiF}_{3}$ we have shown the occurrence of a spin-flop transition.
Its description differs markedly from that of the same transition in uniaxial antiferromagnets because it is due to the reversible displacement of twin-walls. This work also illustrates some difficulties which can be encountered in magnetooptical measurements on cubic antiferromagnets. Such experiments must obviously be undertaken with great care.

We wish to thank Dr. F. Moch for fruitful discussions and we acknowledge the assistance of Mrs. Vernazza (Département de Recherches Physiques, Université Paris VI) who oriented and polished the samples.

\section{References}

[1] JaCOBS, I. S., J. Appl. Phys. 32 (1961) 615.

[2] Dudko, K. L., Eremenko, V. V. and Fridman, V. M., Sov. Phys. JETP 34 (1972) 362.

[3] Millner, A. A., Popkov, Yu. A. and Eremenko, V. V., Sov. Phys. JETP 18 (1973) 20.

[4] King, A. R. and Paquette, D., Phys. Rev. Lett. 30 (1973) 662.

[5] Pisarev, R. V., Ferre, J., Duran, J. and Badoz, J., Solid State Commun. 11 (1972) 913.

[6] Hirakawa, K., Hashimoto, T. and Hirakawa, K., J. Phys. Soc. Japan 16 (1961) 1934.

[7] Slack, G. A., J. Appl. Phys. 31 (1960) 1571.

[8] Néel, L., Proc. of the Kyoto Conf. on theoret. Phys. 1953, Science Council of Japan, Tokyo (1954) 701.

[9] Badoz, J., Billardon, M., Boccara, A. C. and Briat, B., Symp. Faraday Soc. 3 (1969) 27.
[10] Sell, D. D., Greene, R. L. and White, R. M., Phys. Rev. 158 (1967) 489.

[11] Copsey, D. N., Mc Caffery, A. J., Gale, R., Rowe, M. D. and Brint, P., Chem. Phys. Lett. 13 (1972) 533.

[12] Petrov, E. G., Sov. Phys. JeTP 33 (1971) 573.

[13] Pisarev, R. V., Ferre, J., Petit, R. H., Krichevtsov, B. B. and Syrnikov, P. P., J. Phys. C 7 (1974) 4143.

[14] Ferre, J., Pisarev, R. V., Harding, M. J., Badoz, J. and Kizhaev, S. A., J. Phys. C 6 (1973) 1623.

[15] Moch, P. and Dugautier, C., Proc. Int. Conf. Magn., Moscow, 1973.

[16] Griffin, J. A., Schnatterly, S. E., Farge, Y., Regis, M. and Fontana, M. P., Phys. Rev. B 10 (1974) 1960. 